\title{
Enhancing Replacement Policy of Content-Centric Networking to Support Reaction toward Natural Disaster
}

\author{
Raaid Alubady*1 $^{1}$, Samah AbdAlhadi ${ }^{2}$, Wesam Abduladheem Kamil ${ }^{3}$ \\ ${ }^{1}$ Collage of InformationTechnology, University of Babylon, Babylon, Iraq \\ ${ }^{2}$ Collage of Science for Women, University of Babylon, Babylon, Iraq \\ ${ }^{3}$ Computer Science Department, University of Thi-Qar, Thi-Qar, Iraq, \\ *Corresponding Author E-mail: raaid.n.alubady@uobabylon.edu.iq
}

\begin{abstract}
Replacement policy in Content-Centric Networking $(\mathrm{CCN})$ is a necessary and current, function as an important part in Interest packet caching. Pending Interest Table (PIT) is the main and core cache tables in CCN and plays a significant role for recording the information of Interest packets that are forwarded but are still waiting for matching with incoming Data packets. However, PIT management is more fundamental with regard to CCN operations for better memory efficiency. The PIT size determination of the forwarding system is a difficult problem in PIT management. Due to the limited PIT sizing, PIT replacement is utilized to remove the current entry from PIT and constructing a new space for the incoming entry to it. In a disaster area, this problem is due to the massive Interest packet that generating by survivors from the disaster and rescuers. The PIT overflow could be subjected due to use of long Interest lifetimes that would simultaneously increase the number of entries in the PIT. Thus particularly when there is no flexible replacement policy, hence affecting PIT performance. Therefore, the ultimate aim of this paper is to develop the replacement policy that can deal with this problem. The proposed policy is a PIT management based on CCN PIT replacement policy for managing the PIT during a natural disaster, which can lead to mitigating PIT overflowing. The results showed the overall scenarios, the proposed policy better PIT memory usage as well as decreasing the Interest drop, delay time, Interest lifetime and Interest retransmission. A positive significance influence in this work would be to presents a formulate a rule as a function which can decrease the delay and thus be leading to increasing PIT utilization, which will be very much useful for survivors, emergency rescue teams as well as emergency operation centers.
\end{abstract}

Keywords: Content-Centric Networking, Future Internet Architecture, Pending Interest Table, Natural Disaster, Network Simulation.

\section{Introduction}

Content-Centric Networking $(\mathrm{CCN})$ [1][2][3] is an efficient and simple communication model that design based on InformationCentric Networking paradigm for future of the Internet, which completely redesign and develop the Internet by replacing IP with content chunks as universal components of transport. Although there are many studies that have focused on naming, caching, security, and scalability in order to make $\mathrm{CCN}$ getting perfect, nevertheless, the management field; especially in Pending Interest Table (PIT) is still one of the essential concerns of high-speed forwarding. Thus, the management of PIT is one of the most significant design details that have not been studied in the CCN context to a significant extent. Moreover, PIT management is more fundamental with regard to the $\mathrm{CCN}$ operation for better memory efficiency [4][5]. Although it is feasible with the current technology, it leads to suffering from many issues such as PIT lookup [6], PIT Interest lifetime [7], PIT scalability [8] and PIT overflow [5].

According to [9], the management techniques of PIT classified into four categories: PIT implementation, PIT placement, PIT replacement, and adaptive Interest packet lifetime. For cache performance, a replacement policy has a significant influence on it since they are playing an effective and pivotal role in memory management [10]. In respect of increasing the effectiveness of PIT cached, the popular requests are stored at the PIT, which may reduce the redundant traffic that passes through the $\mathrm{CCN}$ networks and it also reduces an average number of access hops.

Communication immediately after a natural disaster situation is an important component of response and recovery, in that it connects the citizen, survivors, emergency operation centers, rescue teams, and support systems [11]. Accessible and reliable communication and network systems also are key to a community's resilience [12][13]. Most probably during the natural disasters, most of the infrastructures are damaged. In this case, without proper networks, communications cannot be established for the area, leading to longer delays in emergency operations that also causing huge losses in human and infrastructures [14][15].

Therefore, the aim behind this paper is that the management of PIT (i.e., content replacement) has not got much attention until now since it is a new novel information structure, which does not appear in any other ICN architecture. In addition, PIT management is challenging because it requires updates for each package, and the requires that is stored in the PIT for a long time require more memory [16]. This is the reason for focusing on the PIT in this paper where PIT plays a significant part in the performance of $\mathrm{CCN}$ routers. Moreover, the current policies that are used with PIT have not considered the popularity of request, entry lifetime and request-hop number as factors for determining the PIT entry which must be replaced.

Thus, leading to believe that these factors are required and may affect the on PIT utilization in disaster area situation. The remainder of the paper has been organized into six sections. Section 2 gives a brief description of CCN, PIT, replacement 
policies and disaster areaon existing works. Propose policy describes in Section 3. Section 4 determines the simulation setup, performance metrics as well. Section 5 presents the results and discussions, and we conclude the paper in Section 6 .

\section{Problem Basic Idea and Related Works}

In this section, explaining the main components as a background, which is related to our proposed model

\subsection{Content-Centric Networking}

Of course, the Internet architecture for the future will not only bring new content and media. It should be aimed to retain some of the current semantics, but with added functionalities of how data would be handled[17]. Also, attention requires to be paid to the privacy policies, presentation, services, communications, and infrastructure, which are essential building constitute of the Internet architecture for the future [18]. Therefore, the new generation of the Internet namely Information-Centric Network (ICN) [1][19][20] introduced a radical change in Internet communications. The various ICN initiatives are focused on designing a new architecture for the future of the Internet that will replace the current host-centric model. Therewith, ICN has gained the interest of the research communities as a new model for networks that can better meet the needs of users in a networked world [21]. A number of designs have emerged in the last few years, including Data-Oriented Network Architecture (DONA), Content-Based Network/Combined Broadcast and Content-Based, Content-Centric Networking (CCN), Network of Information, and others [21][9].

$\mathrm{CCN}$ has attracted much emphasis in the research area recently, with different exploration activities focusing on the rising research with the point of moving from the present Internet engineering which is manufactured and intended for a host to host correspondences model. In addition, $\mathrm{CCN}$ is an efficient and simple communication model driven by subscribers who broadcast requests (i.e., Interest packet) to ask for a content by name regardless of the IP addresses of the nodes that supply the content. Interests' packets are forwarded by intermediate nodes (i.e., CCN router) upstream to publishers that are any node stores or owes the requested content. Publishers simply respond to the Data packets request, which goes through the way back to the subscribers [22][23].

\subsection{Pending Interest Table}

On the context of CCN router design, Pending Interest Table (PIT) represented a core component for forwarding the Interest packet. It is one of the three cache tables newly inculcated into the CCN router designs [4]. Each PIT record comprises five fields [24] as illustrated in Figure 1, including content name (a name related with the entry), incoming faces list, outgoing faces list, entry expire time and forwarding strategy.

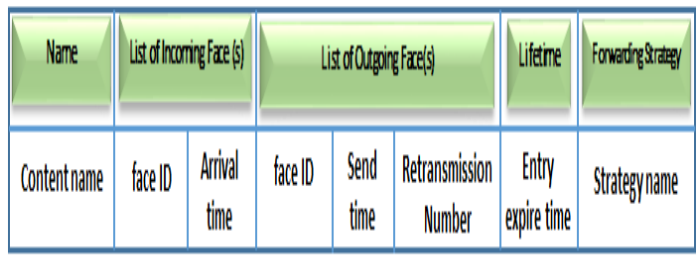

Fig. 1: PIT Entry Fields

Because of the Data packet and Interests packet should exactly match, PIT cannot utilize aggregation. Indeed, PIT requires a large memory for storing the pending entries. Consider a case where the average Interest packets arriving rate is 125 million packets/second and each Interest packet needs $80 \mathrm{~ms}$ for its Round
Trip Time (RTT) [25]. Hence, the current memory technologies are not capable to handle the PIT implementation. On the other hand, the PIT needs to store a number of Interest packet in the order of $10^{7}[26]$. Therefore, huge memories are needed with their known limitations, especially when dealing with their access time. Moreover, PIT in the CCN router is very dynamic. Thus, for all arriving Interest packets and matching Data packets, hence a special process (i.e., lookup process) must be happening in the PIT which must be completed faster. This requires quick memories unfortunately available only for small storage size. Hence, the PIT table may be overflowed. Because PIT receives and removes them exponentially [27].

\subsection{PIT Replacement Policies}

Replacement policy in the PIT is one of the important factors that determine the effectiveness of the cache (e.g., PIT). It has become more significant as technology trends emerge towards highly correlate cache practices [28]. The state-of-the-art procedures, therefore, utilize many of the caches replacement policies, indicating that there is no common replacement that stands out as the best [9]. Hence, this section explores some common PIT replacement policies in greater perspective. It is therefore paramount to investigate the performance of different PIT in relations to replacement policies for a contemporary workload in different PIT configurations. This will determine how some existing policies relate to PIT.

In addition, PIT replacement policy has a different influence on instruction and entries into PIT [29]. In CCN architecture, there are three replacement policies exist, which are Least Recently Used, Random as well as Persistent, and it assumes Persistent as the default replacement policy in CCN [24][30]. Also, a new one is designed and implemented namely Highest Lifetime Least request [29] for this purpose.

\subsection{Natural Disasters Communication}

Over recent years, a number of natural disasters have occurred in the world, causing damage to human, property and almost everything in the area [31]. These disasters of nature like heavy snowfall, floods, and heavy rain (see Figure 2) have always struck at unpredictable times in different places in the world, leading to the increasing losses in human and infrastructure.

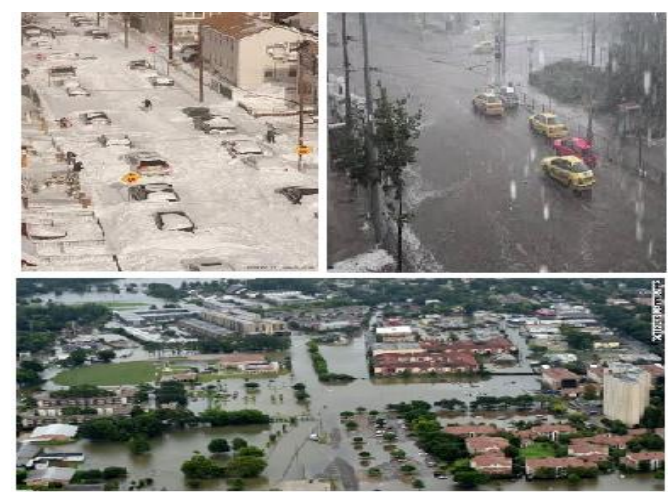

Fig. 2: Example of Natural Disaster

Abdul Hannan at. el., [32] proposed an NDN-IoT-based DMS in an SC scheme in fire disaster environment, which is enabling push support on the fixed sequence number. For that, researchers modified legacy NDN publishes and subscribes functionality with the addition of a threshold limit and fire sensor monitoring modules as well as modified NDN cache tables (particularly FIB and PIT) in disaster scenario for better efficiency.

Reference [33] proposed a framework for flooding disaster scenario in cities and shown the ability of their mechanism (i.e., Smart Threshold Interest Lifetime) in NDN to decrease the packet delay and increase network utilization. Hence, it will be very 
helpful for the rescue teams, survivors, and the emergency operation center.

Enabling communication in a Delay Tolerant Network using Information-Centric Networking is highlighted in Reference [34]. In this work, researchers focus on PULL support-based disaster situations where data mules movement is random and unpredictable.

Baldini at. el., in [35] also designed a fast emergency deployment mobile communication nodes, which are used in many communication technologies to provide multiple communications services in disaster management situations. Also, this work supported multi-media content sharing as well as supported live video streaming along with the services of traditional communication. Another research paper [36] developed a flexible network architecture, which supplies a common networking platform for a performance in emergency's situations and for heterogeneous multiple operator's networks.

\section{Highest Lifetime Lest Request-hop}

One of the effective mechanisms used to manage $\mathrm{CCN}$ routers memory (i.e., CS and PIT) is the replacement policies. Replacement policies of the PIT are useful in expelling entry from the PIT and constructing new location for incoming Interest packets. Due to the limited PIT size, thus, it cannot store all incoming requests. Therefore, by using the replacement policy, PIT will have the ability to monitor incoming flow entry to it and to manage the entries inside it when it is overflowing. Highest Lifetime Least Request [29][37] is a kind of memory management policy, which is utilized to manage and monitor PIT entries within the CCN routers when it is full. This is usable when the PIT is overflowing and then there is a new incoming entry that requires to be added to the PIT. HLLR is replacing an entry in which has a maximum Lifetime value with a minimum number of incoming faces. The policy described as below equation:

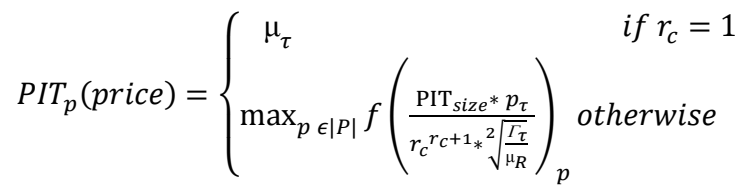

Where $\operatorname{PIT}_{p}$ (price) is denoted as the entry requirement to replace when the PIT overflow. $p_{\tau}$ referred to entry lifetime; $r_{c}$ referred to the frequency of entry already cached into PIT. Whereas the $\mu_{\tau}$ is referred to the maximum PIT entry lifetime. The PIT size is the total number of entries currently belong to PIT; $\Gamma_{\tau}$ is a total amount of the thethelifetime for all entries; $\mu_{R}$ referred is a total amount of requests received from all faces.

In this study, we adaptedthis equation to tackle the issue of PIT overflow, especially, in a disaster area. The reason behind introducing this policy is that to make the ability to optimize the management of the PIT when becoming full because of increasing the request packet on it in a natural disaster environment. This policy is based on HLLR policy as well as requiring to calculate the average number of access hops to requested contents in each time PIT receive a new entry when it's become overflow.

Therefore, in HLLRh policy, the Interest hop count is denoted by $\hbar$, where $\hbar_{a v r}$ is considered as the average number of hop count. Based on that, the Equation 1 is updated as follows:

$$
\begin{aligned}
& \operatorname{PIT}_{p}(\text { price })= \\
& \begin{cases}\max _{p \in|P|} f\left(p_{\tau} * \hbar_{\text {avg }}\right)_{p} & \text { if } r_{c}=1 \\
\max _{p \in|P|} f\left(\left(\frac{\mathrm{PIT}_{\text {size }} p_{\tau}}{r_{c} r_{c}+1^{2} \sqrt{\frac{\Gamma_{\tau}}{\mu_{R}}}}\right) \cdot \hbar_{\text {avg }}\right)_{p} & \text { otherwise }\end{cases}
\end{aligned}
$$

As described above, the Equation (2) has two options for managing PIT entries upon experiencing an overflow. These two options have been discussed next on the basis of their functionality in the context of PIT replacement algorithm.

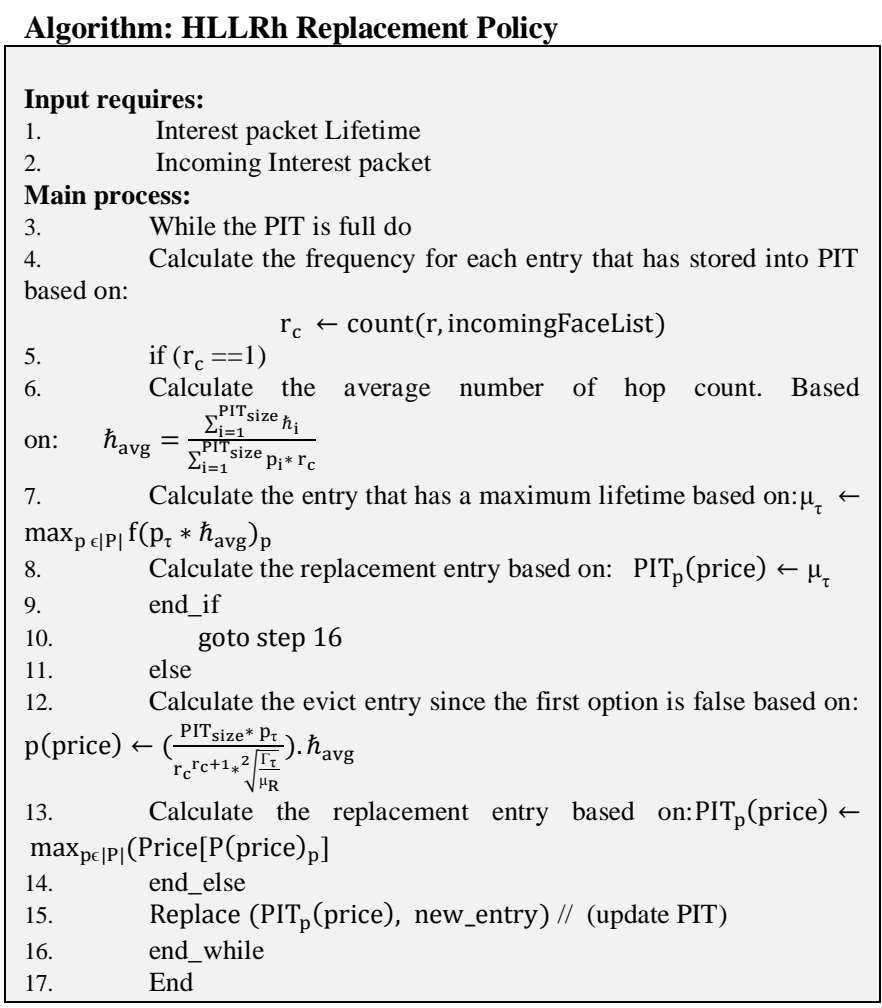

\section{Simulation Setup and Performance Metrics}

\section{- $\quad$ Simulation Environment Selection}

In networks environment, simulation is the first choice of simulating real and dynamic scenarios. It is flexible and reliable tools for studying and evaluating the performance of many protocols [38]. Moreover, according to complex experiments scenarios that ensure correctness of the analysis and evaluation, simulation is considered as the acceptable and widely used method in different research areas, for example, management sciences, operational research, and network system. Several researchers have used this method as a principal technique for reliable publications, i.e., IEEE, ACM, SIGCOMM, INFOCOM, etc. It shows that simulation is a widely accepted method for famous international journals and conferences that make it realistic for the outcomes of the research[39], is a module which can be plugged into the Network Simulator 3 (NS3), which can also support the major aspects of $\mathrm{CCN}$ is called ndnSIM. ndnSIM can be used for the implementation of numerous $\mathrm{CCN}$ applications, for example, the implementation of $\mathrm{CCN}$ routing, caching and forwarding schemes.

\section{- $\quad$ Simulation Parameters}

Particularly, a comprehensive experimental is performed, using a private machine with Intel Core (TM) i73612QM at 16 GBytes of RAM, 3.20 GHz CPU, as well as Linux Ubuntu operating system version 14.04. On the other hand, Table 1 shows the values of the simulation experiment parameters that are commonly used for all the considered scenarios.

Table 1: Simulation Parameters

\begin{tabular}{cc}
\hline Parameters & Value \\
\hline Simulator & NS3-ndnSIM \\
Topology & Rocketfuel-mapped \\
Forward Strategy & Flooding \\
\hline
\end{tabular}




\begin{tabular}{cc}
\hline CS size & 100 Data packet \\
PIT size & 1000 Interest packet \\
Interest Transmission Rate & 1000 to 10000 Interest per second \\
Interest lifetime & $80 \mathrm{~ms}$ \\
Replacement Policy for CS & LRU \\
Replacement Policy for PIT & LRU, Random, Persistent, HLLR \\
Simulation Time & 30 seconds \\
\hline
\end{tabular}

\section{- $\quad$ Evaluation Metrics}

In this study, we compared the performance of our proposed policy with related policies. Therefore, it is important to choose a suitable set of performance metrics. This study focuses on the Interest satisfaction delay, PIT hit ratio, Interest drop, average entry lifetime and Interest retransmission metrics that could be used to measure the performance of the proposed objectives of this study. This performance evaluation will be done by the same metrics that are used by other researchers in the literature [40][41][42][43].

$\checkmark$ Interest Satisfaction Delay: it perceived by the consumer, which measures the waiting time to receive a given content after sending its request.

$\checkmark \quad$ Interest Drop: it can be explained as it is the ratio of lost packets to a total number of sending packets during simulation time.

$\checkmark \quad$ Interest Retransmission: it measures the ratio of the number of Interest retransmissions, which is perceived by the subscribers when there is no response from an upstream node after a period of time.

$\checkmark \quad$ PIT Hit Ratio: it is the ratio of the total number of requested objects found in the PIT.

$\checkmark$ Entry Lifetime: it is referred to the average lifetime of requested objects found in the PIT.

\section{Results and Discussion}

By using NS3-ndnSIM, we evaluate the performance of the HLLRh policy by comparing with another PIT policies. Thus, to guarantee the consistency of the presented results, the HLLRh policy is simulated on the Rocketfuel-mapped topology (see Figure 3). In Rocketfuel-mapped topology[37], nodes are classified into three kinds: $108 \mathrm{CCN}$ routers, 296 subscriber nodes, and 221 publisher nodes. This work intends to analyze the impact of Interest packet rate (i.e., 1000, 2000, 3000, 4000, 5000, $6000,7000,8000,9000$ and 10000 Interest/second) on a performance of PIT as well as on the performance of a network. Therefore, the link delays and link capacities are set as follows: the delays and capacities of $\mathrm{CCN}$ router to $\mathrm{CCN}$ router links are set as $10 \mathrm{~ms}$ and $0.05 \mathrm{Gbps}$, respectively; subscriber node to $\mathrm{CCN}$ router links are set as $10 \mathrm{~ms}$ and $0.1 \mathrm{Gbps}$, respectively; and CCN router to publisher node links are set as $10 \mathrm{~ms}$ and $0.1 \mathrm{Gbps}$, respectively. Moreover, each subscriber node or publisher node is linked to one $\mathrm{CCN}$ router that is selected regularly between those available in the network. Thus, the objective of this section is to evaluate the performance of HLLRh policy relative to current policies for the PIT under various Interest Transmission rate.

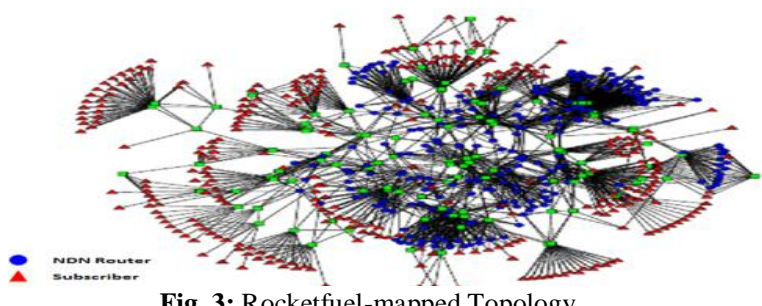

Fig. 3: Rocketfuel-mapped Topology
For each experimental scenarios, the results obtained from the HLLRh policy are compared with related PIT replacement policies results.

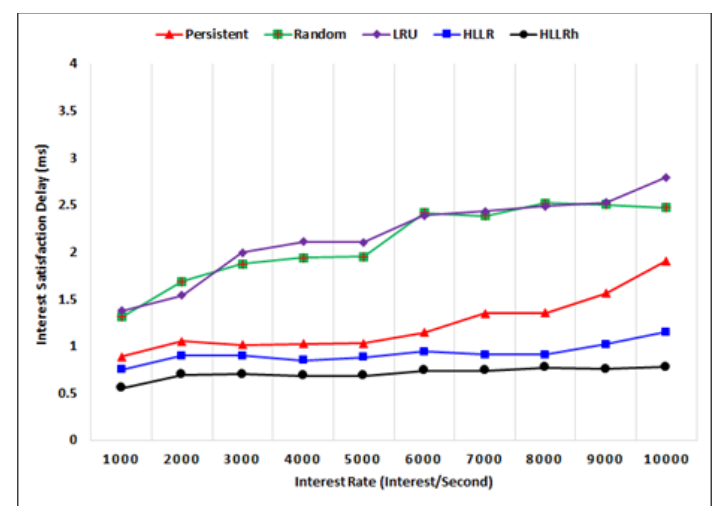

Fig 4: Interest Satisfaction Delay vs Interest Rate for PIT Policies

Figure 4, results is related to Interest Satisfaction Delay. The scattering results clearly display that the satisfaction delay at each traditional PIT policies is influenced more by the increasing Interest rate, except HLLR and HLLRh. The satisfaction delay succeeded by experimental measurement scenario for HLLRh is $0.549 \mathrm{~ms}$ at the Interest rate is 1000 packet/second, and slightly increases to $0.774 \mathrm{~ms}$ at the Interest rate are $10000 \mathrm{packet} / \mathrm{second}$. The result of other policies is $0.7498 \mathrm{~ms}$ and $1.147 \mathrm{~ms}$ at the Interest rate1000 and 10000 packet/second, respectively, to HLLR as well as the LRU is higher that had a $1.373 \mathrm{~ms}$ and $2.792 \mathrm{~ms}$ at the Interest rate1000 and 10000 packet/second, successively. By contrast, both Random and Persistent policies have shown high satisfaction delay of $1.3102 \mathrm{~ms}$ and $2.468 \mathrm{~ms} ; 0.8886 \mathrm{~ms}$ and $1.907 \mathrm{~ms}$ at the Interest rate1000 and 10000 packet/second, respectively. As such, the HLLRh policy provides better/lowest Interest Satisfaction Delay performance than HLLR, LRU, Random, and Persistent.

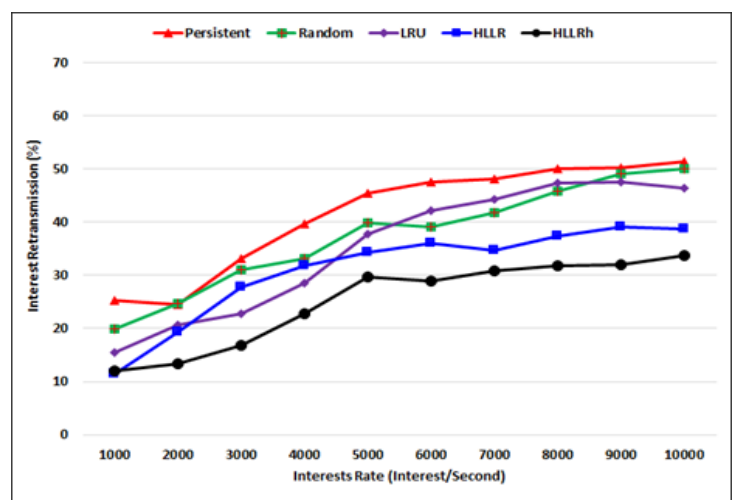

Fig. 5: Interest Retransmission vs Interest Rate for PIT Policies

Figure 5 depicts the results of Interest retransmission for all five policies when the Interest rate is between 1000 to 10000 Interest/second. The show results it is obvious that Interest retransmission of the HLLRh is $12.009 \%$, HLLR is $11.39 \%$, LRU is $15.555 \%$, Random is 19.903 , and Persistent is $25.273 \%$ at the Interest rate 1000 Interest/second, respectively.Also, according to the results obtained, it can be seen for the HLLRh, HLLR, LRU, Random, and Persistent, the Interest retransmissions is increased as Interest packet rate increases, this percentage is achieved as $33.63 \%, 38.769 \%, 46.29 \%, 49.99 \%$ and $51.3777 \%$, respectively, at Interest rate 10000 Interest/second. Remarkably, the performance gap between the HLLRh with the closest policy (HLLR) result is achieved $13.04 \%$ at Interest rate 10000 Interest/second. Hence, the results revealed that HLLRh again has provided the lowest Interest retransmission than the other PIT policies. 


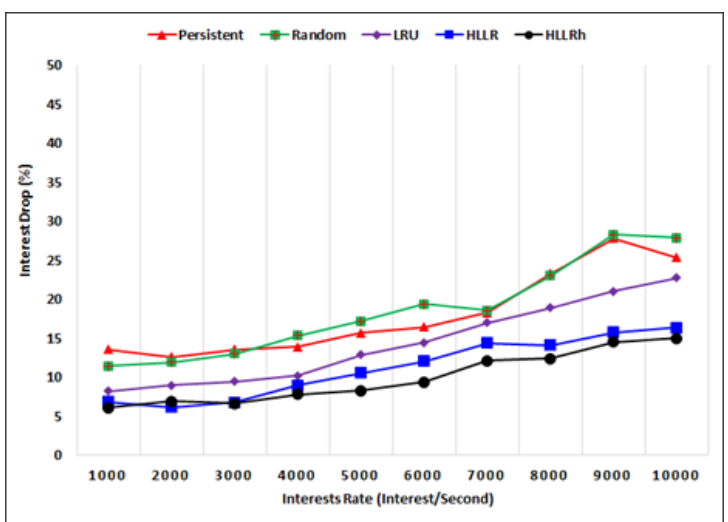

Fig. 6: Interest Drop vs Interest Rate for PIT Policies

While Figure 6 explains the deployment of our proposed (HLLRh) policy for PIT replacement of various Interest rates. In a typical scenario, PIT is the basic structure used to keep the state of each active flow. However, the size of the PIT is a critical challenge since because it is feasible with the current technology, it leads to suffering from many issues, for example, PIT overflow. According to the result showed that Persistent, Random, and LRU provided the highest Interest drop value of $13.55 \%, 11.442 \%$, and $8.203 \%$ at Interest rate 1000 packet/second, respectively. Whereas Random, Persistent, and LRU Interest packet rate is 10000 Interest/second, the Interest drop increases gradually to become $27.908 \%, 25.35 \%$, and $22.734 \%$, successively. As for HLLR policy, the results of Interest drop performance of $6.845 \%$ and $16.38 \%$ at Interest rate 1000 and 10000 Interest/second. This is because HLLR has an ability to evict only the entry that has the less frequent and highest entry lifetime. As saw in the given figure, when the Interest rate is setting 1000 Interest/second, the result obtained by HLLRh is $6.11 \%$. Similarly, when the Interest rate is setting 10000 Interest/second, the result obtained by HLLRh is $14.99 \%$. Therefore, it can be possible to conclude that HLLRh can serve as a good replacement technique.

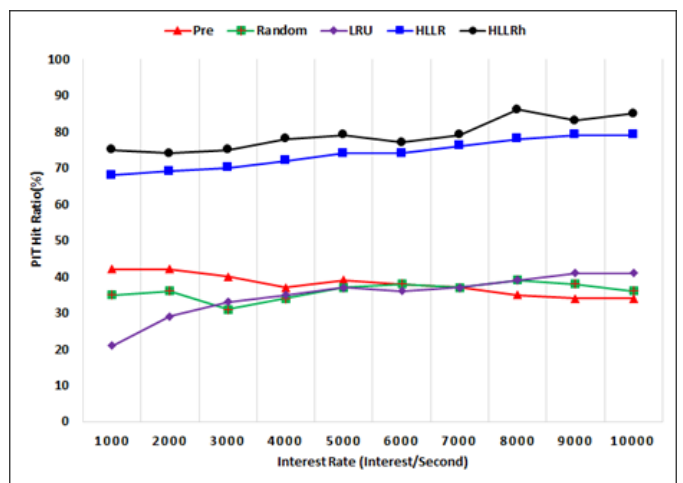

Fig. 7: PIT Hit Ratio vs Interest Rate for PIT Policies

PIT hit ratio is used to provide a good insight into the network caching performance when the Interest packets are shared in a constant manner based on the size of the PITs. Figure 7 illustrates the average PIT hit ratio of all five PIT replacement policies when the Interest rate is between 1000 to 10000 Interest/second. HLLRh and HLLR have the highest value for the PIT hit ratio of $75.838 \%$ and $68.1003 \%$, respectively, at Interest rate are 1000 Interest/second as compared to the other three policies (Persistent is $42.326 \%$, Random is $35.51 \%$ and LRU is $21.309 \%$ ). In a similar way, both HLLRh and HLLR again they have obtained the same behavior as a result since the Interest packet rate increases.

The average PIT hit ratio also increases incremental, which is achieved $85.53 \%$ and $79.098 \%$, respectively, with an Interest packet rate are setting as 10000 Interest/second. However, HLLRh increased the average PIT hit ratio value from $51.46 \%$ to $67.65 \%$ at Interest rate 1000 to 10000 Interest/second, respectively.

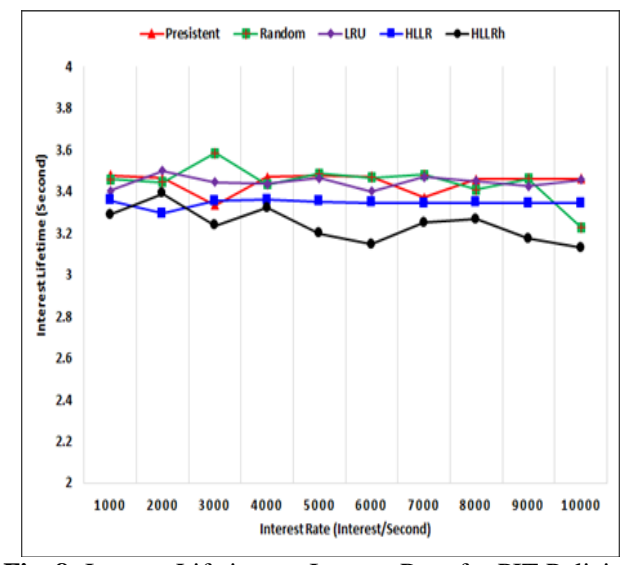

Fig. 8: Interest Lifetime vs Interest Rate for PIT Policies

Figure 8 displays the average Interest lifetime of HLLRh, HLLR, LRU, Random, and Persistent policies with the given Interest rate between 1000 to 10000 Interest/second. From the figure above, it can be noticed clearly that all policies oscillate their values up and down between 3.583 seconds and 3.144 seconds. However, it is obvious during all experiments that the average Interest lifetime is reduced when HLLRh policy is used, while the Persistent and Random policies give the highest average Interest lifetime. Meanwhile, it can be also noticed that the HLLR policy maintained its performance and the changing values are slightly when increasing the Interest rate between 3000 to 10000 Interest/second. In addition, the HLLRh policy continues to give the lowest results; in the case of Interest rate 10000 Interest/second, its value for the average Interest lifetime is lower than $0.101 \%, 0.104 \%, 0.10808 \%$, and $0.10827 \%$ to Random, HLLR, LRU, and Persistent, respectively.

In brief, HLLRh, HLLR, LRU, Random, and Persistent policies were compared in the Rocketfuel-mapped topology, and the results are presented in Figures 4-8. The analysis shows that HLLRh. HLLR, LRU, Random, and Persistent operate similarly in dealing with the PIT conditions and various Interest rate. HLLRh achieves a higher PIT hit ratio rate when compared with other PIT policies. Moreover, HLLRh reduces significantly the Interest satisfaction delay, Interest lifetime, Interest retransmission, and Interest drop, experienced by the Interest packet rate.

\section{Conclusion}

Since CCN is still in its infancy in terms of standardization. Therefore, the researchers attempted to submit this study as an experimentally proven contribution to help in the future draft and provide a design for the Internet of the future. In this paper, a new PIT replacement policy within $\mathrm{CCN}$ routers is proposed and evaluated. The HLLRh uses Interest lifetime, Interest frequency, and an average number of hops for Interest in order to determine which entry inside PIT will be evicted when the PIT become full. Hence, allowing the new entry to be in. In addition, the current research also presented a highly simulated empirical comparison between the proposed policies with different PIT replacement management policies in CCN. The study demonstrated the unequivocal presentation of CCN's leading research communities in adopting the HLLRh policy as the best replacement policy for CCNs that can be utilized in a natural disaster environment. The study, therefore, found that the HLLRh replacement policy is of great importance to the CCN's performance in terms of reducing Interest satisfaction delay, Interest drop, entry lifetime, and Interest retransmission rate, as well as a better hit ratio with wide percentage against the others. Since that policy, has the ability to manage and monitor the PIT under various Interest rates. The proposal for the future is to extend the simulation run to other traffic generation platform and other different network topology. 


\section{References}

[1] A. Morelli, M. Tortonesi, C. Stefanelli, and N. Suri, "InformationCentric Networking in Next-generation Communications Scenarios,” J. Netw. Comput. Appl., vol. 80, pp. 232--250, 2016.

[2] F. Oehlmann, "Content-Centric Networking," Netw. Archit. Serv., vol. 43, pp. 11-18, 2013.

[3] D. Saxena, V. Raychoudhury, S. Neeraj, C. Becker, and J. Cao, "Named Data Networking: A Survey," Comput. Sci. Rev. Elsevier, vol. 19, pp. 15--55, 2016.

[4] A. J. Abu, B. Bensaou, and J. M. Wang, "Interest Packets Retransmission in Lossy CCN Networks and its Impact on Network Performance," in Proceedings of the 1st international conference on Information-centric networking - INC '14, 2014, pp. 167-176.

[5] M. Virgilio, M. Guido, and S. Riccardo, "PIT Overload Analysis in Content Centric Networks," in Proceedings of the 3rd ACM SIGCOMM workshop on Information-centric networking, 2013, pp. 67-72.

[6] V. Matteo, P. Diego, and L. Linguaglossa, "On the Design and Implementation of a wire-speed Pending Interest Table," in Computer Communications Workshops (INFOCOM WKSHPS), 2013 IEEE Conference on, 2013, vol. 13, pp. 369--374.

[7] S. H. Bouk, S. H. Ahmed, M. A. Yaqub, D. Kim, and M. Gerla, "DPEL: Dynamic PIT Entry Lifetime in Vehicular Named Data Networks," IEEE Commun. Lett., vol. 20, no. 2, pp. 336--339, 2016

[8] G. Carofiglio, M. Gallo, D. Perino, and L. Muscariello, "Pending Interest Table Sizing in Named Data Networking," in Proceedings of the 2nd International Conference on Information-Centric Networking, 2015, pp. 49-58.

[9] R. Alubady, "An Efficient Pending Interest Table Control Management in Named Data Network," Universiti Utara Malaysia (UUM), 2017.

[10] D. Grund and J. Reineke, "Estimating the Performance of Cache Replacement Policies," in Proceedings - 6th ACM and IEEE International Conference on Formal Methods and Models for CoDesign, MEMOCODE'08, 2008, pp. 101-111.

[11] R. Nijenhuis, "The International Environmental Emergencies Response System: A Case Study of Supertyphoon Haiyan (Yolanda), The Philippines," Asian J. Environ. Disaster Manag., vol. 6 , no. 2 , pp. 175-190, 2014

[12] A. M. Al-Bakry and A. K. Hadi, "Collaborative Computer Aid Diagnosis Framework in Cloud Environment based on Mult Agents Systems," Int. J. Adv. Eng. Technol., vol. 7, no. 1, p. 21, 2014.

[13] V. G. Menon, J. P. Pathrose, and J. Priya, "Ensuring Reliable Communication in Disaster Recovery Operations with Reliable Routing Technique," Mob. Inf. Syst., vol. 2016, pp. 1-11, 2016

[14] A. K. Idrees, "Neural Network for QoS Multicast Routing in Computer Networks,” J. Babylon Univ., vol. 18, no. 3, 2010.

[15] L. Bhimarao and S. Uma, "Disaster Area Communication Using Neighbor coverage in MANET," Int. J. Sci. Eng. Technol. Res., vol. 3, no. 5, pp. 1310-1314, 2014

[16] Z. Li, K. Liu, Y. Zhao, and Y. Ma, "MaPIT : An Enhanced Pending Interest Table for NDN With Mapping Bloom Filter," IEEE Commun. Lett., vol. 18, no. 11, pp. 1915-1918, 2014

[17] S. Hassan, A. Habbal, R. Alubady, and M. Salman, "A Taxonomy of Information-Centric Networking Architectures based on Data Routing and Name Resolution Approaches," J. Telecommun. Electron. Comput. Eng., vol. 8, no. 10, 2016.

[18] W. Yang and Y. Qin, "An Interest Shaping Mechanism in NDN Joint Congestion Control and Traffic Management," in 2018 IEEE International Conference on Communications (ICC), 2018, pp. 1-6.

[19] M. Tortelli, D. Rossi, G. Boggia, and L. A. Grieco, "ICN Software Tools: Survey and Cross-Comparison," Simul. Model. Pract. Theory - Elsevier, vol. 63, pp. 23-46, 2016

[20] G. M. Brito, P. B. Velloso, and I. M. Moraes, Information-Centric Networks A New Paradigm for the Internet, 1st ed. Croydon, Surrey CR0 4YY: John Wiley $1 \&$ Sons, 2013.

[21] X. Wang, J. Lv, M. Huang, K. Li, J. Li, and K. Ren, "Energyefficient ICN Routing Mechanism with QoS Support," Comput Networks, vol. 131, no. December 2017, pp. 38-51, 2018

[22] X. Q. Xdqj et al., "Scalable High-Speed NDN Name Lookup," in Proceedings of the 2018 Symposium on Architectures for Networking and Communications Systems, 2018, pp. 55-65.

[23] D. Saxena and V. Raychoudhury, "Radient: Scalable, Memory Efficient Name Lookup Algorithm for Named Data Networking," J. Netw. Comput. Appl., vol. 63, pp. 1-13, 2016
[24] A. Afanasyev, I. Moiseenko, and L. Zhang, "ndnSIM: NDN Simulator for NS-3," University of California, Los Angeles, Technical Report NDN-0005, California, Los Angeles, pp. 1-7, 2012.

[25] D. Perino, B. Labs, B. D. Styles, and C. Network, "A Reality Check for Content Centric Networking," in ACM ICN 2011, 2011, pp. 4449.

[26] W. You, B. Mathieu, P. Truong, J.-F. Peltier, and G. Simon, "DiPIT: A Distributed Bloom-Filter Based PIT Table for CCN Nodes," in 2012 21st International Conference on Computer Communications and Networks (ICCCN), 2012, pp. 1-7.

[27] H. Dai, B. Liu, Y. Chen, and Y. Wang, "On Pending Interest Table in Named Data Networking," in Proceedings of the eighth ACM/IEEE symposium on Architectures for networking and communications systems - ANCS '12, 2012, pp. 211-222.

[28] S. J. Taher, O. Ghazali, and S. Hassan, "A Review on Cache Replacement Strategies in Named Data Network," J. Telecommun. Electron. Comput. Eng., vol. 10, no. 2-4, pp. 53-57, 2018

[29] R. Alubady, S. Hassan, and A. Habbal, "HLLR : Highest Lifetime Least Request Policy for High Performance Pending Interest Table," in 2016 IEEE Conference on Open Systems (ICOS), October 10-12, 2016, Langkawi, Malaysia HLLR:, 2016, pp. 42 47.

[30] S. Hassan, R. Alubady, and A. Habbal, "Performance Evaluation of the Replacement Policies for Pending Interest Table," J. Telecommun. Electron. Comput. Eng., vol. 8, no. 10, pp. 125-131, 2016.

[31] T. Gomes et al., "A Survey of Strategies for Communication Networks to Protect against Large-scale Natural Disasters," in Proceedings of 2016 8th International Workshop on Resilient Networks Design and Modeling, RNDM 2016, 2016, pp. 11-22.

[32] A. Hannan et al., "Disaster Management System Aided by Named Data Network of Things: Architecture, Design, and Analysis," Sensors, vol. 18, no. 8, pp. 1-20, 2018.

[33] R. Alubady, S. Hassan, and A. Habbal, "Adaptive Interest Lifetime in Named Data Networking to Support Disaster Area," J. Telecommun. Electron. Comput. Eng., vol. 10, no. 2-4, 2018.

[34] J. Seedorf, D. Kutscher, and B. S. Gill, "Decentralised Interest Counter Aggregation for ICN in Disaster Scenarios," in Globecom Workshops (GC Wkshps), 2016 IEEE, 2016, pp. 1-6.

[35] G. Baldini, S. Karanasios, D. Allen, and F. Vergari, "Survey of Wireless Communication Technologies for Public Safety," IEEE Commun. Surv. Tutorials, vol. 16, no. 2, pp. 619-641, 2014.

[36] A. G. Fragkiadakis, I. G. Askoxylakis, E. Z. Tragos, and C. V Verikoukis, "Ubiquitous Robust Communications for Emergency Response Using Multi-Operator Heterogeneous Networks,' EURASIP J. Wirel. Commun. Netw., vol. 2011, no. 1, p. 13, 2011.

[37] R. Alubady, S. Hassan, and A. Habbal, "Pending Interest Table Control Management in Named Data Network," J. Netw. Comput. Appl., vol. 111, pp. 99-116, 2018.

[38] A. Ud Din, Ikram and Hassan, Suhaidi and Habbal, "A Content Placement Scheme for Information-Centric Networking," Adv. Sci. Lett., vol. 21, no. 11, pp. 3482--3484, 2015

[39] S. Mastorakis, A. Afanasyev, I. Moiseenko, and L. Zhang, "ndnSIM 2 . 0: A New Version of the NDN Simulator for NS-3," University of California, Los Angeles, Tech. Rep. NDN-0028, California, Los Angeles, pp. 1-8, 2015.

[40] S. H. Ahmed, S. H. Bouk, M. A. Yaqub, D. Kim, H. Song, and J. Lloret, "CODIE: Controlled Data and Interest Evaluation in Vehicular Named Data Networks," IEEE Trans. Veh. Technol., vol. 65 , no. 6, pp. 3954-3963, 2016

[41] G. Carofiglio, M. Gallo, L. Muscariello, and M. Papalini, "Multipath Congestion Control in Content-Centric Networks," in Computer Communications Workshops (INFOCOM WKSHPS), 2013 IEEE Conference on, 2013, pp. 363-368.

[42] H. Choi, J. Yoo, T. Chung, N. Choi, T. Kwon, and Y. Choi, "CoRC: Coordinated Routing and Caching for Named Data Networking," in Proceedings of the tenth ACM/IEEE symposium on Architectures for networking and communications systems ANCS '14, 2014, pp. 161-172.

[43] Y. Sun et al., "Trace-Driven Analysis of ICN Caching Algorithms on Video-on-Demand Workloads," in Proceedings of the 10th ACM International on Conference on emerging Networking Experiments and Technologies - CoNEXT '14, 2014, pp. 363-376. 